\title{
A note on the empirics of the neoclassical growth model
}

\author{
Giovanni Caggiano* \\ Leone Leonida \\ University of Glasgow \\ Queen Mary, University of London
}

\begin{abstract}
This paper shows that the widely used log-linearization of the neoclassical model of growth implies a relevant loss in terms of the ability of the model in replicating the patterns of convergence of an economy to its equilibrium level.
\end{abstract}

Keywords: AutoCorrelation Function; Convergence processes; Neoclassical growth model.

$J E L: \mathrm{C} 22 ; \mathrm{N} 10 ; \mathrm{O} 40$

\footnotetext{
${ }^{*}$ Corresponding author. Department of Economics, University of Glasgow, Glasgow, G12 8RT, UK. Tel.: +44-141-3305059; fax: +44-141-3304940. E-mail address: g.caggiano@socsci.gla.ac.uk
} 


\section{Introduction}

Since Mankiw, Romer and Weil (1992) and Barro and Sala-i-Martin (1992), it is common in the empirical literature to employ a log-linearized version of the neoclassical model of growth to test for convergence.

However, although it provides a closed-form solution of the model and a link between theory and the empirical specification to be estimated, there exists a growing dissatisfaction about the use of log-linearization. For example, Reiss (2000) suggests that it implies an error whose magnitude depends on the returns to scale and on the distance of the economy from its steady state position. Dowrick (2004) shows that the log-linearization of the model is unable to produce a linear specification in the variables, which questions whether it is worth to linearize the model at all, since linear techniques cannot anyway be applied to estimate the structural parameters of interest.

We propose a formal testing procedure to evaluate the empirical performance of the loglinearized version of the neoclassical model in replicating convergence patterns. We approximate the actual convergence pattern of the economy to its steady state by the AutoCorrelation Function (ACF, henceforth) of detrended real GDP per capita and test the null hypothesis that it is not statistically different from the ACF implied by the log-linearized version of the neoclassical model. Our results suggest that the latter cannot replicate the transitional dynamics of 14 OECD economies out of the 15 we examine, the only exception being the U.S. economy. At the very least, this implies that the log-linearization reduces dramatically the ability of the model of replicating the features displayed by the data.

The paper proceeds as follows. Section 2 describes our procedure. Section 3 reports our results, and Section 4 concludes. 


\section{Transitional dynamics in the linearized neoclassical growth model}

The ACF of a time series process is a measure of the correlation of the series with its past. The analysis of its shape provides us with information about the rate at which shocks vanish. If we assume that a deterministic linear trend is a proxy for the equilibrium path of the economy, the ACF of detrended real GDP per capita would then measure the transitional dynamic pattern of the economy towards its steady state path.

Since a model that does not give rise to an ACF like that observed in the data is misspecified (see Abadir and Talmain, 2002), we can assess the empirical validity of the neoclassical growth model by testing whether the ACF implied by its log-linearized version can replicate the persistence pattern shown by the empirical ACFs of detrended real GDP per capita.

To recover the theoretical ACF, consider an economy where saving decisions and technological progress are exogenous. Assume that total production follows a Cobb-Douglas production function, and that technical progress is labor augmenting:

$$
Y_{t}=K_{t}^{\alpha}\left(A_{t} L_{t}\right)^{1-\alpha}
$$

where $Y_{t}, K_{t}, L_{t}, A_{t}$, are output, capital, labor and A is the level of technology, all measured at time $t$, with $0<\alpha<1$. A and $L$ are assumed to grow exogenously at rate $g$ and $n$, respectively. A constant fraction of output, $s$, is invested. Moreover, by defining $\hat{k}=K / A L$ and $\hat{y}=Y / A L$ as the stock of capital and output per effective unit of labor, the evolution of $\hat{k}$ can be described by the following expression:

$$
\frac{d \hat{k}_{t}}{d t}=s \hat{k}_{t}^{\alpha}-(n+\delta+g) \hat{k}_{t}
$$

where $\delta$ is the depreciation rate. Under the hypothesis of diminishing returns to capital, 
equation (2) implies that $\hat{k}_{t}$ converges towards its steady state level $\hat{k}_{t}^{*}$ :

$$
\hat{k}^{*}=\left(\frac{s}{n+\delta+g}\right)^{1 /(1-\alpha)}
$$

Consider then a log-linear approximation of equation (3) around the steady state:

$$
\frac{d\left[\ln \left(\hat{y}_{t}\right)\right]}{d t}=-\beta\left[\ln \left(\hat{y}_{t}\right)-\ln \left(\hat{y}_{t}^{*}\right)\right]
$$

where $\hat{y}_{t}^{*}=\left(\hat{k}_{t}^{*}\right)^{\alpha}$ and $\beta=(1-\alpha)(n+\delta+g)$.

As common in the time series literature on growth, Michelacci and Zaffaroni (2000) suggest to test for convergence process by discretizing (4):

$$
y_{i, t}-y_{i, t-1}=g_{i}+\beta_{i} y_{i, t-1}^{*}-\beta_{i} y_{i, t-1}
$$

or, by allowing for the Jones invariance property, which implies that $y_{i, t}^{*}=g_{i} t+y_{i, 0}$ is (the log of) the level of real per capita output in the steady state:

$$
y_{i, t}-y_{i, t-1}^{*}=\left(1-\beta_{i}\right)\left(y_{i, t-1}-y_{i, t-1}^{*}\right)
$$

where $y_{i, t}$ is the $\log$ of real per capita output in country $i, y_{i, t}=\ln \left(Y_{i, t} / L_{i, t}\right)$.

Equation (6) is an $\mathrm{AR}(1)$ process whose theoretical $\mathrm{ACF}$ is:

$$
\tilde{\rho}_{i}(\tau)=\left(1-\beta_{i}\right)^{\tau}
$$

Hence, to test whether the ACF implied by (6) can replicate the pattern displayed by the empirical ACFs of detrended real GDP per capita, we fit (7) to the data by using the first $k$ realizations of the empirical ACF of $\left\{y_{i, t}-y_{i, t}^{*}\right\},{ }^{1}$ and test the null hypothesis that the fitted and the actual ACFs are statistically different:

$$
H_{0}: \tilde{\rho}(\tau)=\rho_{i}(\tau), \quad \forall \tau \quad \text { vs } \quad H_{A}: \tilde{\rho}(\tau) \neq \rho_{i}(\tau), \quad \text { for at least one } \tau
$$

\footnotetext{
${ }^{1}$ Since the estimation of the tail of the empirical ACF is likely to be imprecise, we discard $1 / 4$ of the observations (Box and Jenkins, 1976).
} 
If the null hypothesis is rejected, we conclude that the model is unable to replicate the convergence process of that particular economy to its equilibrium level.

\section{Data and empirical results}

We use data on real GDP per capita for 15 OECD countries - Australia, Austria, Belgium, Canada, Denmark, Finland, France, Germany, Italy, Japan, Netherlands, Norway, Sweden, UK and USA - over the period 1900-2000. We use the Penn World Table (MARK 6.1) and extend up to 2000 the annual log real GDP per capita series provided by Bernard and Durlauf (1995) - using 1987 as base year to splice the data.

It is important to stress that our testing procedure requires a robust inference setting for the ACF. In the case of stationary series, standard theory provides asymptotically correct confidence bands, but for nonstationary series neither exact nor asymptotic results are available. Because of the lack of theoretical results, we use resampling techniques for dependent data to construct robust confidence bands around the ACF of $\left\{y_{i, t}-y_{i, t}^{*}\right\}$.

To our knowledge, the only resampling technique that provides asymptotically valid confidence bands for the autocorrelations of a potentially nonstationary process is subsampling (see Politis, Romano and Wolf, 2004). However, subsampling provides confidence bands for $k<T$ lags only. In order to construct confidence bands for the entire sample, we propose to use the subsampling confidence bands as a benchmark to which compare other resampling methods for dependent data and then choose the one that minimizes a distance function with the subsampling confidence intervals. More precisely, let $\mathbf{c}_{1 j}$ and $\mathbf{c}_{2 j}$, with $j=1, \ldots, k$, denote the confidence bands for the first $k$ autocorrelations estimated by subsampling. Let $\widehat{\mathbf{c}}_{1 j}^{h}$ and $\widehat{\mathbf{c}}_{2 j}^{h}$ be the confidence bands estimated by using technique $h=1, \ldots, H$. Let $\mathbf{e}^{h}=\left(\widehat{\mathbf{c}}^{h}-\mathbf{c}\right)$, 
where $\mathbf{c}=\left[\mathbf{c}_{1 j} \mid \mathbf{c}_{2 j}\right]$ and $\widehat{\mathbf{c}}^{h}=\left[\widehat{\mathbf{c}}_{1 j}^{h} \mid \widehat{\mathbf{c}}_{2 j}^{h}\right]$ are the $(2 \times k)$ matrices obtained by horizontally concatenating the two confidence bands. The optimal confidence interval, $\widehat{\mathbf{c}}^{h_{O P T}}$, will be such that

$$
\widehat{\mathbf{c}}^{h O P T}=\arg \min F(e)
$$

where $F(e)=\sum_{i=1}^{2} \sum_{j=1}^{k}\left|\widehat{\mathbf{c}}_{i, j}^{h}-\mathbf{c}_{i, j}\right|$.

In our empirical exercise, the $95 \%$ confidence bands have been constructed via the stationary bootstrap (Politis and Romano, 1994), which minimized the proposed criterion for all the series. ${ }^{2}$

Figure 1 shows our results for three cases, representative of different transitional dynamics patterns: U.S., Italy and the UK.

\section{$<$ Insert Figure 1 here $>$}

The empirical results suggest that the log-linearized version of the neoclassical model can replicate the pattern of the empirical ACF of the U.S. economy - the $95 \%$ confidence bands constructed around the empirical ACF contain the theoretical one. In the case of Italy and UK, instead, the fitted ACF is not contained into the confidence bands: the null hypothesis of equality between the fitted and the actual ACFs must be rejected.

Figure 2 shows that the null hypothesis must also be rejected for the remaining cases.

\section{$<$ Insert Figure 2 here $>$}

\footnotetext{
${ }^{2}$ We have also considered are the moving block bootstrap (Kunsch, 1989), the tapered block bootstrap (Paparoditis and Politis, 2001) and the sieve bootstrap (Buhlmann, 1997).
} 
If anything, these findings cast doubts on the claim that the log-linearized version of the neoclassical growth model is a general framework to describe convergence processes; rather, the transitional dynamics implied by the Solow model seems to replicate the U.S. convergence pattern only.

\section{Concluding remarks}

We show that the log-linearized version of the neoclassical growth model is unable to replicate the transitional dynamics of real GDP per capita to its equilibrium path for 14 out of the 15 OECD economies we examined. Our findings suggest that the dynamics implied by such simplified version of the model is not rich enough to replicate the observed patterns. Rather, it is able to explain the U.S. convergence process only - possibly, because such economy is close to its steady state path. At the very least, this result implies that the use of log-linearization to solve macroeconomic models, although a useful device, implies a dramatic loss in terms of the ability of replicating the observed dynamics of economic variables.

In this perspective, our results add to the existing dissatisfaction towards such version of the neoclassical model of growth. However, in our opinion they also open the question on whether any model predicting convergence to the steady state may replicate actual convergence processes. Clearly, this issue goes beyond the scope of this note, and is left for future research. 


\section{References}

[1] Abadir, K. M. and G. Talmain, 2002, Aggregation, persistence and volatility in a macro model, Review of Economic Studies 69, 749-779.

[2] Barro, R. J. and X. Sala-i-Martin, 1992, Convergence, Journal of Political Economy 100, 223-251.

[3] Bernard, A. and S. N. Durlauf, 1995, Convergence in International Output, Journal of Applied Econometrics 10, 97-108.

[4] Box, G. P. and G. M. Jenkins, 1976, Time Series Analysis: Forecasting and Control, revised ed., San Francisco: Holden Day.

[5] Buhlmann, P., 1997, Sieve bootstrap for time series, Bernoulli 3, 123-148.

[6] Dowrick, S., 2004, Delinearizing the Neoclassical Convergence Model, in: S. Dowrick, R. Pitchford and S. J. Turnovsky, eds., Economic Growth and Macroeconomic Dynamics: recent development in economic theory, Cambridge, UK: Cambridge University Press, $83-94$

[7] Mankiw, N., Romer, D. and D. Weil, 1992, A Contribution to the Empirics of Economic Growth, Quarterly Journal of Economics 107, 407-437.

[8] Michelacci, C. and P. Zaffaroni, 2000, (Fractional) beta convergence, Journal of Monetary Economics 45, 129-153.

[9] Kunsch, H. R., 1989, The jackknife and the bootstrap for general stationary observations, Annals of Statistics 17, 1217-1241. 
[10] Paparoditis, E. and D. N. Politis, 2001, Tapered block bootstrap, Biometrika 88, 11051119.

[11] Politis, D. N. and J. P. Romano, 1994, The Stationary Bootstrap, Journal of the American Statistical Association 89, 1303-1313.

[12] Politis, D.N., Romano, J.P., and M. Wolf, 2004, Inference for autocorrelations in the possible presence of a unit root, Journal of Time Series Analysis 25, 251-63.

[13] Reiss, J. P., 2000, On the Convergence Speed in Growth Models, Faculty of Economics \& Management Magdeburg (FEMM) Working Paper No. 22/2000. 
Figure 1

Fitted and Empirical ACF - U.S., Italy and U.K.

The solid and the dashed lines represent the empirical and the the fitted ACF. The dashesd-dotted lines represent the 95\% confidence bands.

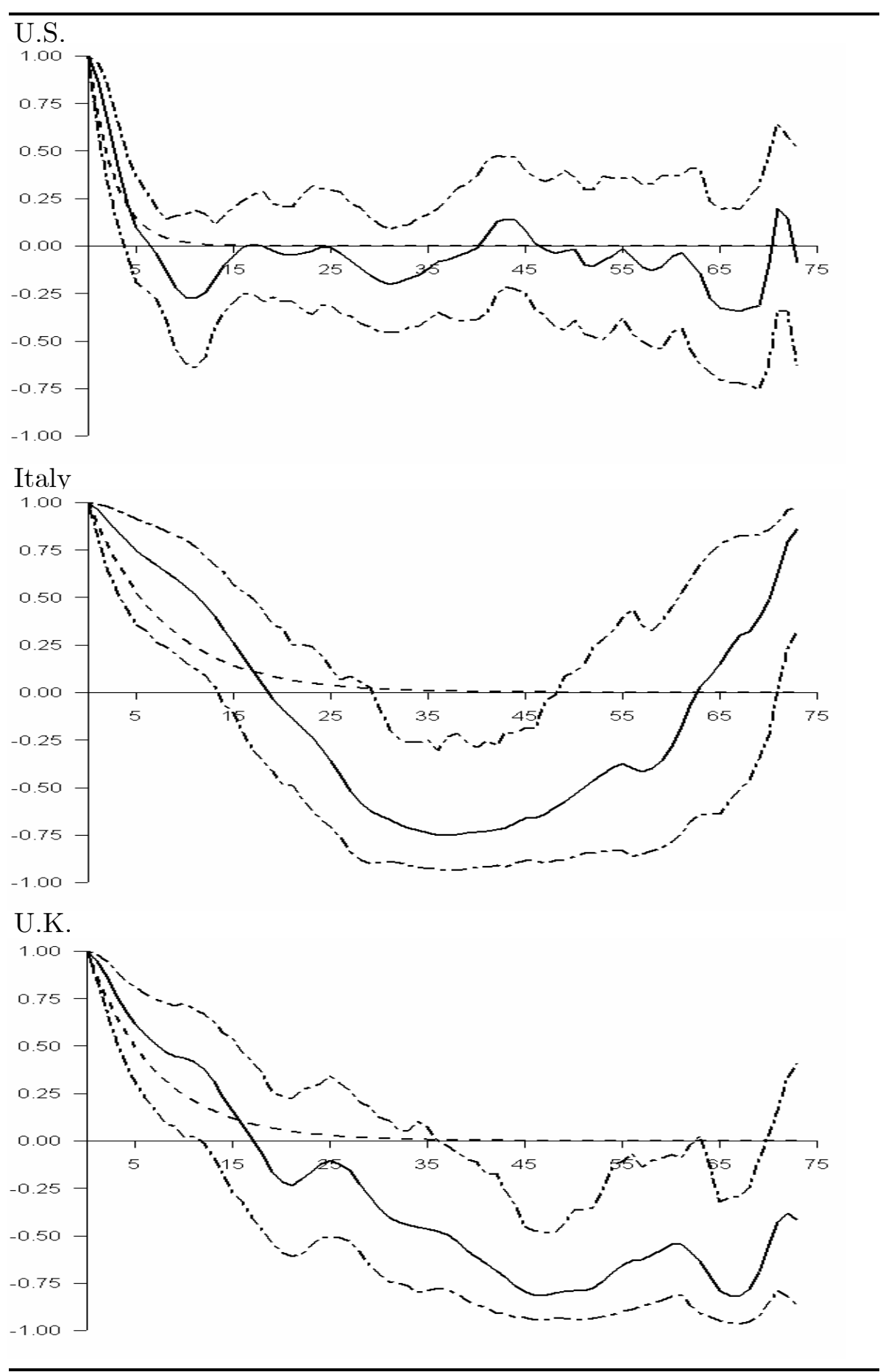


Figure 2

Fitted and Empirical ACF - Remaining Economies

The solid and the dashed lines represent the empirical and the the fitted ACF. The dashesd-dotted lines represent the $95 \%$ confidence bands.
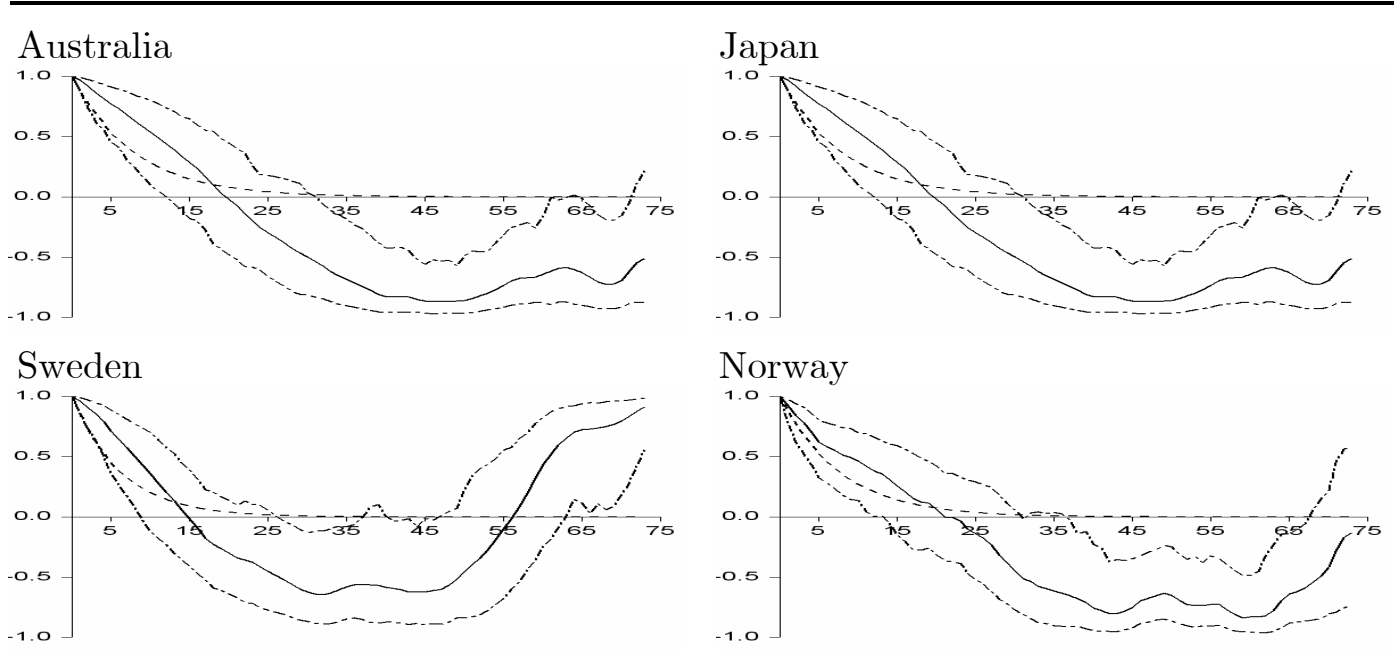

Netherlands

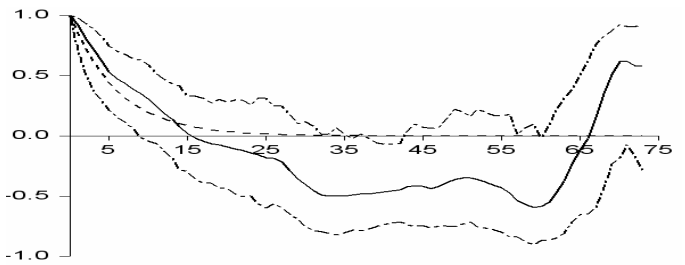

Austria

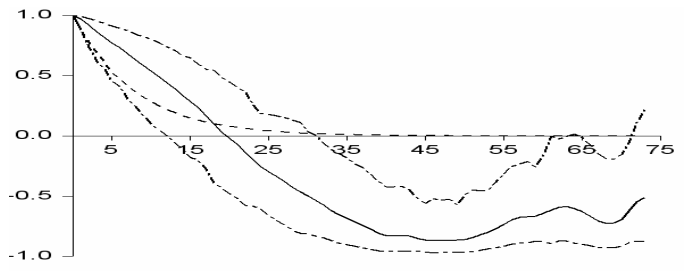

Germany

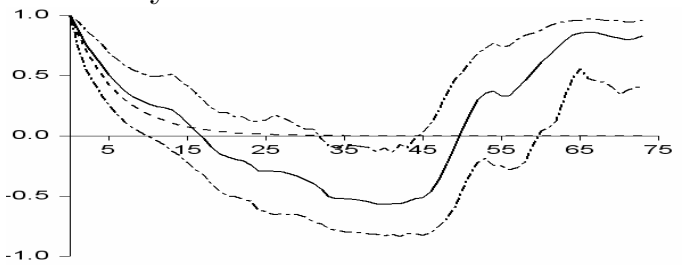

France

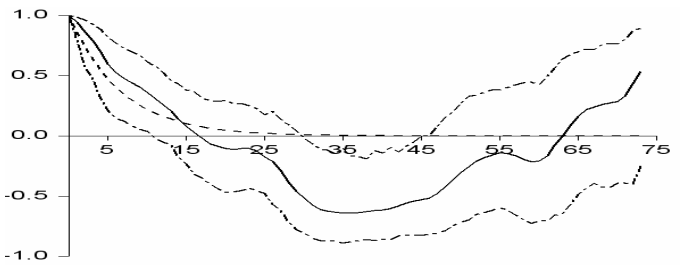

Finland

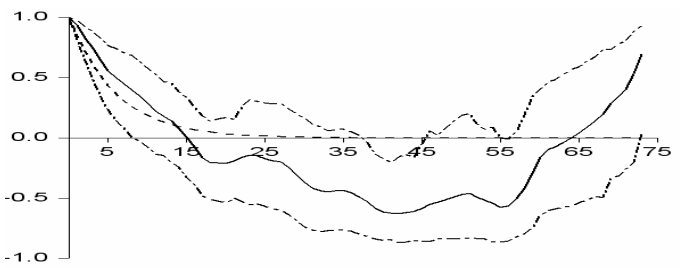

Denmark

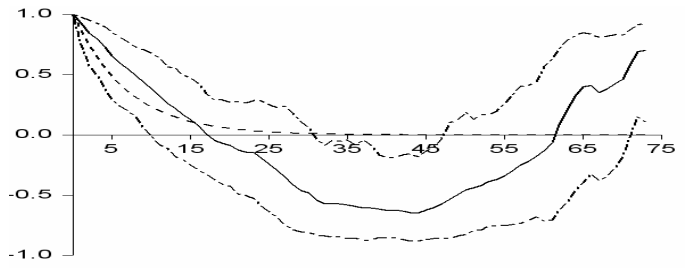

Canada

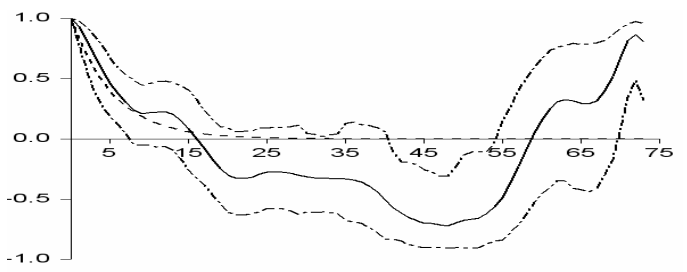

Belgium

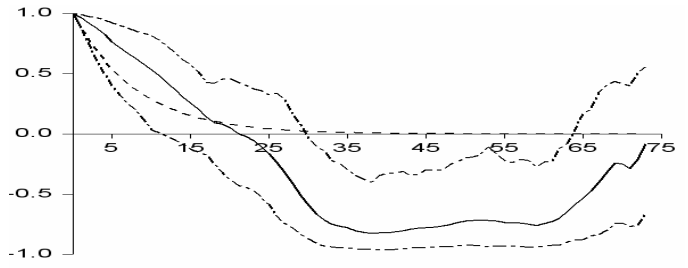

